D.O.I.: $10.3895 /$ gi.v12n4.5211

\title{
USABILIDADE DAS TECNOLOGIAS DE INFORMAÇÃO E COMUNICAÇÃO NO SISTEMA BRT-DF - UMA ANÁLISE CONSTRUTIVISTA DA PERCEPÇÃO DO USUÁRIO
}

\section{USABILITY OF INFORMATION TECHNOLOGY AND COMMUNICATION SYSTEM BRT- DF - AN ANALYSIS CONSTRUCTIVIST USER PERCEPTION}

\author{
Fernanda Santos Lima; Evaldo Cesar Cavalcante Rodrigues²; Roberto Bernardo da Silva ${ }^{3}$; Carlos \\ Rosano Peña ${ }^{4}$ \\ ${ }^{1}$ Universidade de Brasília, UNB - Brasília/DF - Brasil \\ fslima26@gmail.com \\ ${ }^{2}$ Universidade de Brasília, UNB - Brasília/DF - Brasil \\ evaldocesar@unb.br \\ ${ }^{3}$ Universidade de Brasília, UNB - Brasília/DF - Brasil \\ rbaccioly@gmail.com \\ ${ }^{4}$ Universidade de Brasília, UNB - Brasília/DF - Brasil \\ gmcrosano@gmail.com
}

\begin{abstract}
Resumo
O sistema de transportes urbano é considerado um serviço de caráter elementar para a população, pois garante ao usuário o acesso a mobilidade. O sistema Bus Rapid Transit (BRT) tem se mostrado uma alternativa mais vantajosa, frente a outros sistemas de transporte, devido à menor complexidade e custo de implantação. As Tecnologias de Informação e Comunicação (TIC), nesse sentido, desempenham um papel de destaque para que, não apenas o BRT, mas qualquer sistema de transportes alcance maiores níveis de eficiência na prestação de serviço. Desse modo, este estudo buscou analisar a percepção dos usuários em relação às TIC utilizadas no sistema BRT Sul - que atende as regiões administrativas de Santa Maria, Gama e Park Way - a partir da análise multicritério, na qual se pretendeu verificar a influência dessas para a compreensão da usabilidadelqualidade do serviço prestado. Como resultado, verificou-se que os usuários consideram sua utilização de relevância para a qualidade do serviço, de modo que o critério conforto foi considerado o principal elemento de impacto para usabilidade do serviço enquanto que, aprendizado e interface obtiveram desempenhos inferiores.
\end{abstract}

Palavras-chave: usabilidade; tecnologia da informação e comunicação; BRT.

\section{Introdução}

A expansão dos centros urbanos estabelece uma condição de descentralização territorial, já que grande parte da mão de obra empregada reside em áreas mais afastadas, sendo a dependência da utilização do sistema de transporte urbano algo inevitável. Nesse sentido, questões relacionadas à 
mobilidade ganham maior relevância já que tal discussão resgata a noção de aspectos que impactam diretamente na qualidade de vida de uma população.

O sistema de transportes urbano é desse modo considerado um serviço de caráter elementar para a população, já que tem por responsabilidade permitir ao usuário o acesso a mobilidade de modo que seja capaz de deslocar-se livremente por determinado espaço territorial.

Pensar tal questão resgata ainda a noção de uma crescente necessidade do usuário de transportes públicos quanto ao acesso à informação. Nessa lógica, a criação de interfaces de comunicação (TIC) entre transporte e usuário tem por intenção primal auxiliá-lo no processo de orientação e decisão.

O sistema BRT (Bus Rapid Transit) tem se mostrado uma solução para problemas de mobilidade, já que sua implantação é favorecida tendo em vista as relações custo benefício além de demandar menor tempo e complexidade no processo de implantação, se comparado a outros sistemas, como o metroviário, por exemplo, (NTU, 2009).

A utilização de tais dispositivos é considerada de grande relevância nesse processo para que o BRT atinja maiores níveis de eficiência em termos de custo, confiabilidade, conforto e segurança pois tem por objetivo prestar informações ao usuário por meio de seus mecanismos que por definição tendem a ser mais eficientes.

Este trabalho buscou analisar a percepção dos usuários em relação às TIC - Sistemas de Monitoramentos de Imagens, Sistemas de Mensagens Dinâmicas e Sistemas de Sonorização Ambiente nos terminais de passageiros e, principalmente, nos ônibus, utilizadas no sistema BRT Sul, análise na qual se pretendeu verificar a influência dessas percepções para a compreensão da usabilidade/qualidade do serviço prestado.

\section{Ergonomia Cognitiva e Usabilidade}

Segundo Rodrigues (2014), os debates acerca de usabilidade ganham maior relevância a partir da década de 1970, quando estudos relacionados ao desenvolvimento de softwares tornaramse mais consistentes. Andrade (2007) afirma que suas origens estão diretamente vinculadas a Ciência Cognitiva e, até 1980, utilizava-se o termo user-friendly (amigável) para denominar sistemas eficientes no quesito atendimento às necessidades do usuário.

Nesse contexto, admite-se o papel da Ergonomia Cognitiva enquanto campo de aplicação da ergonomia que, segundo Abrahão et al., (2005), remete ao estudo dos processos cognitivos frente a conjunturas de diferentes níveis de complexidade. Seu papel é investigar as formas de manifestação da cognição humana, de modo a ponderar habilidades e limitações, sejam estas de natureza física ou cognitiva, já que tais elementos exercem impacto direto sobre a percepção do usuário em relação aos mecanismos de usabilidade. 
Ainda de acordo com Cybis et. al., (2010), conceitualmente o termo usabilidade estaria ligado à relação estabelecida em um ciclo conexo: usuário, tarefa, interface do software, equipamento e outros elementos presentes no ambiente que o cerca. Desse modo, para tornar possível o estabelecimento de padrões básicos de avaliação capazes de mensurar níveis de usabilidade, é necessário considerar a relação existente entre homem e máquina.

Nesse sentido, Jordan (1998) considera este um dos pontos centrais no que tange ao processo de criação de um produto/serviço voltado para consumo. Considera ainda a existência de uma relação quanto à percepção do usuário - ao utilizar determinado produto/serviço - vinculado ao nível de eficiência para atingir determinado objetivo. Quanto menor essa percepção, menor o índice de satisfação. Além disso, o usuário demonstra uma tendência em associar a questão da facilidade de utilização como sinônimo de qualidade do produto, o que tem despertado o interesse das organizações, já que essa pode ser considerada uma boa oportunidade para o estabelecimento de vantagem competitiva.

De acordo com Ferris (2011), a usabilidade no transporte público é percebida por meio de mensagens sonoras e visuais, mapas táteis para orientação e treinamento dos funcionários do transporte, tendo em vista capacitá-los para auxiliar e informar os usuários.

Quanto ao caráter subjetivo do conceito, Andrade (2007) ressalta a possibilidade de divergências entre usuários/grupos de usuários em relação à compreensão sobre usabilidade. Uma ferramenta pode ser desenvolvida para atender a determinado público e, de fato, cumprir com o pretendido, mas, ao mesmo tempo, demonstrar-se inadequado para outro grupo que esteja inserido em outra lógica, cujos objetivos demonstram-se de maneira divergente. Cybis et. al., (2010) associam essa subjetividade à sensação de bem-estar do usuário em relação à utilização do sistema.

\section{Tecnologias da Informação e Comunicação (TIC)}

Berdichevsky e Neunschwander (1999) estabelecem uma diferenciação no modo como as tecnologias interagem com o ser humano classificando-as como passivas ou ativas. As tecnologias passivas são aquelas que apresentam, de modo geral, a capacidade de informar utilizando-se, sobretudo, de processos de comunicação em massa e servem a fins sociais, políticos e econômicos como jornais, rádio e televisão, por exemplo. Já as tecnologias ativas - web sites, agentes de interface - caracterizam-se por mídias interativas que podem servir a mesma finalidade, diferenciando-se na questão da personalização, simulação e controle, que são aparentemente exercidos pelo expectador.

Nesse aspecto, Rodrigues (2014) afirma que as TIC estão essencialmente ligadas a uma nova forma de comunicação, cujo surgimento pode ser percebido a partir da Terceira Revolução 
Industrial e seu desenvolvimento torna-se mais evidente na década de 1970, recebendo maior destaque em meados dos anos 1990.

Flores et al., (2012) afirma que essas novas formas de comunicação podem ser expressas como produção de ferramentas - hardwares, softwares - utilizadas no processamento de informações, desde a aquisição até o armazenamento, divulgação e aplicação.

Silva et al., (2015) sustenta que, quando associadas a transportes, se referem ao desenvolvimento de dispositivos que em geral estão associados a inclusão de serviços que contribuem para o melhoramento do processo de mobilidade do usuário. De modo geral, os sistemas inteligentes de transportes, segundo Kanninen (1996), são utilizados essencialmente para garantir os aumentos da mobilidade e eficiência no uso das vias, além da redução do tempo de espera, por exemplo.

\subsection{Transporte Público}

A sociedade - sobretudo a população urbana - encontra-se elencada territorialmente à moradia, ao local de trabalho e, esporadicamente, ao lazer. Para Villaça (1989), o homem urbano rotineiramente acaba por ter a necessidade de realizar cada vez mais longos trajetos e, por conseguinte, a dependência de transporte público é ainda maior.

Barat e Batista (1973) definem a função do transporte como meio de integração de áreas urbanas - do aspecto econômico, social e espacial. Costa (2007) afirma que um dos objetivos se refere ao deslocamento do usuário no menor tempo possível, de modo a conferir-lhe maior nível de bem-estar. Araújo et al., (1997) sustenta o caráter positivo quanto à utilização de transporte coletivo, de modo que se tende a gerar considerável redução das filas em congestionamentos. Auxilia ainda na redução dos níveis de poluição, tendo em vista a redução da quantidade de veículos em circulação.

O crescimento desordenado das cidades, de acordo com Sampaio et al., (2006), configura um problema quanto ao fornecimento desse serviço, uma vez que uma rede de transportes coletivo, em suma, é desenvolvida numa tentativa de suprimir uma demanda emergente, não sendo estrategicamente planejado para atender a uma demanda maior que a prevista.

\subsection{Sistema BRT (Bus Rapid Transit)}

O sistema BRT, de acordo com Reis e Machado (2013), é uma modalidade de transporte viável nas grandes cidades e tem como princípio a eliminação de todo e qualquer obstáculo possível na via - como caminhões, veículos de passeio e outros que não fazem parte do sistema - de modo a proporcionar sua exclusividade de locomoção. É definido ainda, conforme Levinson et al., (2003), 
como um modo rápido de transporte resultante de uma combinação da qualidade do transporte sobre trilhos e flexibilidade do transporte rodoviário.

Nessa perspectiva, uma das vantagens apontadas, quanto à utilização, se refere ao grau de efetividade na eliminação dos chamados 'fatores problemáticos' - como a presença de pedestres e cruzamentos de veículos - de modo a favorecer a redução dos índices de incidência de acidentes e aumentar a fluidez e celeridade do transporte.

Segundo o Ministério das Cidades (2009), outra vantagem, quanto à implementação, referese ao grau de flexibilidade, no sentido de que mudanças no sistema podem ser realizadas com investimentos razoáveis em termos de custo e tempo. Além disso, existe a possibilidade de conversão de um corredor BRT para uma alternativa ferroviária, que pode representar, por exemplo, a melhoria de condições financeiras do local ou crescimento da demanda de passageiros.

\subsubsection{Sistema BRT Distrito Federal - Expresso DF (Eixo Sul)}

De acordo com o Plano Diretor de Transporte Urbano e Mobilidade do Distrito Federal e Entorno (PDTU, 2010), o Expresso DF Eixo Sul é responsável por transportar aproximadamente 272 mil pessoas e tem por objetivo a realização de interlocução entre as regiões administrativas e o centro. Abrange as regiões administrativas de Santa Maria, Gama e Park Way. Os corredores exclusivos têm $43 \mathrm{~km}$ de extensão onde os veículos trafegam livremente (exceto os $6 \mathrm{~km}$ do eixo rodoviário), operando em linhas semiexpressas e as tarifas são cobradas antes do embarque, por utilização de cartões recarregáveis nos postos de atendimento localizados nas próprias estações.

Ainda de acordo com o PDTU (2010), o sistema conta com uma frota de 100 veículos sendo 38 Padron e 62 articulados - cuja capacidade de transporte é de até 130 passageiros por viagem. Ao longo do percurso, existem 2 terminais, 10 estações para embarque/desembarque em nível e 10 passarelas, tendo em vista promover maior segurança aos usuários. O projeto contempla ainda a integração aos terminais e estações fechadas com pagamento antecipado, seja no primeiro ônibus ou em bilheterias - estações/terminais.

Segundo o BRT Brasil (2016), um dos principais benefícios, quanto à utilização do sistema, refere-se ao tempo de viagem - 90 minutos - que foi reduzido para uma média de 40 minutos. A proposta do Expresso DF Eixo Sul prevê ainda a utilização de veículos híbridos e elétricos, visto que o projeto trata também da proposição de soluções a questões relacionadas à emissão de gases poluentes. Os ônibus serão monitorados por um Centro de Controle Operacional (CCO), que têm por responsabilidade transmitir aos usuários informações precisas por meio da utilização de painéis eletrônicos. 


\section{Método Multicritério Construtivista (MCDA-C)}

O Modelo Multicriteria Decision Aid Construtivist (MCDA-C) rompe com a visão tradicional de Pesquisa Operacional, que estabelece por base a existência de um único ponto ótimo e traz uma nova perspectiva pautada na identificação de multiresultados esperados.

Bana e Costa (1990) afirmam que, diferentemente de um padrão monocritério - que estabelece a construção de apenas um critério de análise para capturar todos os aspectos relevantes do problema -, este modelo tem por principal característica determinar uma multiplicidade de eixos de análise, no qual vários atores de decisão atuam no processo de definição de parâmetros de análise.

De acordo com Rodrigues (2014), este método divide-se em três etapas: sistematização do modelo, avaliação dos grupos e conclusões finais. Assim, o modelo apresentado foi definido de acordo com a consideração de juízos de valor dos representantes, coleta de dados realizada em contato com os usuários e as considerações finais baseada na análise de resultados obtidos por meio da aplicação do método.

\section{Método de Pesquisa}

A pesquisa foi desenvolvida ao longo do primeiro semestre de 2016 como Trabalho de Conclusão de Curso (TCC) de graduação em Administração pela Universidade de Brasília, seguindo as seguintes etapas: revisão de literatura; definição de critérios; coleta e análise de dados, realizada nos dias 02 e 03 de julho.

\subsection{Descrição da Pesquisa}

Este estudo pode ser compreendido como uma análise de desempenho da utilização de TIC no sistema BRT Sul de Brasília, tendo em vista a perspectiva do usuário. Nesse aspecto, o delineamento da pesquisa apresenta caráter descritivo que, segundo Zanella (2009), expõe como principal preocupação a descrição de fenômenos por meio dos significados transmitidos pelo ambiente. Nesse sentido, o estudo retrata uma abordagem qualitativa e quantitativa, cujos dados foram coletados por meio de aplicação de questionários semiestruturados a uma pequena amostra composta por 31 respondentes.

Conforme Oliveira (2007), o método qualitativo pode ser definido como o detalhamento do estudo de determinado fenômeno, objeto, atores sociais, entre outros; tem por preocupação capturar o significado das informações adquiridas por meio da aplicação de questionários e entrevistas, sem a realização de uma mensuração quantitativa dessas características ou comportamentos. Já a abordagem tem por finalidade a quantificação de dados obtidos por meio das informações coletadas, o que permite maior precisão no que se refere à definição de correlação entre variáveis. Uma crítica 
apontada por Richardson (1999) se refere à herança positivista pautada na separação de fatos e contextos que deveriam ser analisados em conjunto. O reflexo disso é a possibilidade de interpretações falhas ou enviesadas.

Para o desenvolvimento deste estudo, utilizou-se da metodologia de análise multicritério Multicriteria Decision Aid (MCDA) -, em seu aspecto construtivista (MCDA-C), que traz em sua essência a necessidade de participação de todos os envolvidos no processo, tendo em vista a estruturação de uma modelagem matemática para apoio à decisão.

Cabe, nesse sentido, salientar a importância da participação dos especialistas da área de Transportes da Universidade de Brasília (UnB), sobretudo para o processo de construção dos elementos de análise por meio do modelo MCDA-C. O método tem por objetivo prestar suporte ao decisor no processo de tomada de decisão - considerar diferentes opções - levando-se em consideração as concepções de diferentes atores envolvidos no processo que sofrerão de alguma forma o impacto resultante dessas escolhas.

Os instrumentos foram desenvolvidos por meio da realização de Brainstorming (tempestade de ideias) - em duas reuniões com especialistas da área de Transportes da UnB. Nesses encontros, os especialistas contribuíram no processo de definição dos critérios (os chamados Elementos Primários de Avaliação (EPA's)), subcritérios, descritores - presentes na estruturação do roteiro de formulário -, bem como as taxas de contribuição (pesos de cada um dos elementos avaliados para a percepção de usabilidade/qualidade das TIC no sistema BRT na perspectiva do usuário). Nesse aspecto, Yager (1998) afirma que a partir dessa definição de pesos é possível verificar e analisar possíveis situações de risco/relevância na realização de um investimento.

A primeira etapa de elaboração consistiu na definição do rótulo: 'Usabilidade das TIC no sistema BRT - Eixo Sul - do Distrito Federal na perspectiva dos usuários'. Ensslin et al.; (2001) afirma que a principal função do rótulo se refere a delimitação do campo de análise, de modo que é possível identificar quais os itens mais relevantes para o atingimento de resolução do problema.

Os atores envolvidos são classificados como: intervenientes (participação direta nas etapas do processo decisório); os agidos (atores com participação passiva no processo de tomada de decisões, mas que são diretamente afetados pelos resultados advindos desse processo); decisores (responsáveis pelo sistema de transportes) e os representantes (especialistas na área de Transportes). Após a realização das reuniões com os atores envolvidos e a validação dos EPA's, foi possível o estabelecimento dos Pontos de Vista Fundamentais considerados de maior significado para o processo decisório, conforme demonstra o Quadro 1. 
Quadro 1- Pontos de Vista Fundamentais

\begin{tabular}{|l|l|}
\hline $\begin{array}{l}\text { Pontos de Vista } \\
\text { Fundamentais (PVF's) }\end{array}$ & Detalhamento do Critério direcionado à ação \\
\hline Aprendizado & $\begin{array}{l}\text { Shackel (1991) define que o aprendizado une a facilidade de compreensão e } \\
\text { retenção a médio ou longo prazo das informações pelos clientes. }\end{array}$ \\
\hline Interface & $\begin{array}{l}\text { Apresentar layout amigável, ou seja, de fácil entendimento quanto à transmissão da } \\
\text { informação para o usuário. }\end{array}$ \\
\hline Movimentação do usuário & $\begin{array}{l}\text { Assegurar ao usuário a possibilidade de deslocamento em menor tempo possível, } \\
\text { tendo em vista sua infraestrutura (organização, horários e rotas). }\end{array}$ \\
\hline Percepção da marca BRT & $\begin{array}{l}\text { Reflete o modo como o indivíduo percebe a satisfação de suas necessidades - } \\
\text { sejam estas físicas, sociais ou psicológicas. }\end{array}$ \\
\hline
\end{tabular}

Fonte: Autoria própria (2016).

Após a definição dos critérios de análise, foi realizada uma decomposição de cada elemento em vários subcritérios, tendo em vista a identificação de ações potenciais. Foram ainda definidos os descritores, cuja finalidade se ateve em apresentar da melhor forma os aspectos considerados pelos decisores - de modo a considerar, por exemplo, categorias de preferência, verificar desempenho de ações relacionadas ao critério e ordenar um modelo de avaliação coeso.

\subsection{Participantes do Estudo}

Os participantes do processo de construção do modelo foram quatro professores vinculados ao Departamento de Administração da Universidade de Brasília (UnB); dois doutorandos - nas áreas de Administração e Transportes respectivamente, um mestrando em Transportes e um funcionário do Transporte Urbano do Distrito Federal (DFTRANS) bacharel em Ciências Contábeis -; e cinco alunos graduandos em Administração. Desse modo, assumem o papel de representantes dos decisores, no qual, por meio da realização da técnica de Brainstorming (tempestade de ideias), buscou-se a construção de critérios relevantes para a percepção de cada um dos integrantes com relação à usabilidade das TIC no sistema BRT - Eixo Sul - DF.

\subsection{Coleta e análise de dados}

Para fins de coleta de dados, utilizou-se da aplicação de questionários estruturados aos usuários do sistema BRT-DF no qual os entrevistados responderam as questões nas quais a escala de classificação apresentou cinco opções estabelecidas, a fỉm de avaliar o grau de significância da utilização das TIC na perspectiva dos usuários, conforme o Quadro 2. 
Quadro 2 - Descrição da classificação de respostas dos usuários

\begin{tabular}{|c|c|c|}
\hline Classificação & Expectativa & Descrição \\
\hline Muito significante & Acima das Expectativas & Muito relevante para usabilidade do serviço. \\
\hline Significante & \multirow{2}{*}{ Dentro das Expectativas } & Relevante para a usabilidade do serviço. \\
& & Pouco relevante para a usabilidade do serviço. \\
\hline Pndiferente ou Neutro & Ausência de Expectativas & Indiferente para a usabilidade do serviço. \\
\hline Gera incômodo & Abaixo das Expectativas & Gera incômodo para a usabilidade do serviço. \\
\hline
\end{tabular}

Fonte: Autoria própria (2016).

Pretendeu-se observar, desse modo, a percepção de qualidade do usuário quanto à qualidade do serviço de informação neste sistema de transportes.

\section{Análise de desempenho das TIC no sistema BRT Sul na percepção do usuário}

A identificação da percepção do usuário se baseou em quatro grandes critérios de análise: Aprendizado, Interface, Conforto (movimentação do usuário) e Valor da Marca BRT, que serão analisados nos tópicos a seguir.

Para melhor compreensão, cabe salientar quanto aos gráficos apresentados nesta seção que esses têm por finalidade demonstrar o desempenho das TIC em relação a cada critério de análise. A linha superior traz o desempenho máximo de usabilidade, enquanto a linha inferior denota o desempenho mínimo; já a linha intermediária demonstra os resultados obtidos a partir da percepção do usuário.

\subsection{Aprendizado}

Neste critério os questionamentos direcionados aos usuários foram baseados em dois subcritérios: 'Funcionalidade' - aspectos relacionados à percepção de facilidade de utilização das TIC - e 'Compreensão', no qual o enfoque esteve na percepção de uso, por exemplo, da forma como as informações são transmitidas, bem como da percepção de ocorrência de erros no sistema.

Nesse aspecto, Shackel (1991) define a questão do aprendizado como a união entre facilidade de compreensão e retenção das informações em médio ou longo prazo pelos clientes/usuários, conforme demonstra a Figura 1. 
Figura 1 - Resultado do critério 'Aprendizado'

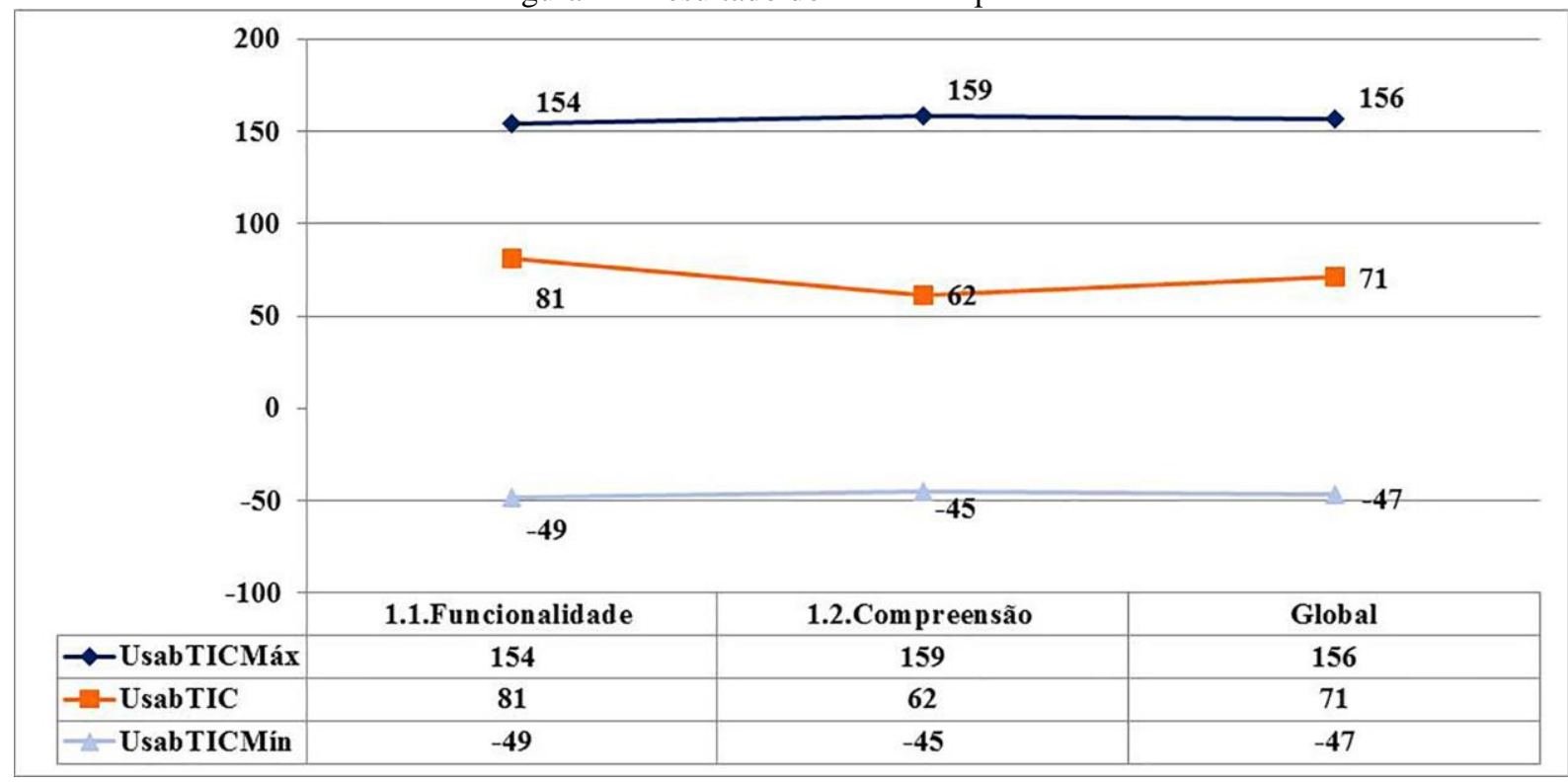

Fonte: Autoria própria (2016).

Dentre os subcritérios analisados, a questão da 'Funcionalidade' foi mais bem avaliada pelos usuários, com desempenho igual a 81, apresentando uma diferença de 73 pontos em relação ao desempenho máximo observado. Quanto ao item 'Compreensão', verificou-se que este obteve pior classificação na categoria, apresentando uma diferença de 97 pontos entre a avaliação de desempenho realizada pelos usuários e o nível de desempenho máximo. Isso significa que, dentre os subcritérios analisados, a questão da 'Funcionalidade' demandaria um esforço menor de investimento para implementação de melhorias, já que o nível de qualidade percebido pelo usuário está mais próximo do ponto máximo de desempenho.

Partindo-se de uma análise em perspectiva global, no critério 'Aprendizado' observou-se uma diferença de 203 em relação à distância dos pontos mínimo e máximo de desempenho. A qualidade percebida pelos usuários foi percebida acima no ponto acima do nível mínimo - igual a 71 -, enquanto o nível máximo estaria em 156. Embora este aspecto seja classificado de modo geral como 'significante' - relevante para o desempenho da usabilidade do serviço -, seria necessária uma melhoria em torno de 45,5\% para um atingimento máximo de desempenho.

\subsection{Interface}

De acordo com Ferreira (1999), o conceito de interface estaria associado ao conjunto de elementos de hardware e software que possuem finalidade de possibilitar interação com o usuário.

No que tange aos subcritérios 'Layout' e 'Comunicabilidade', as questões foram direcionadas ao aspecto físico das TIC, nos quais se verificou a percepção sobre como essas foram desenvolvidas, atentando-se ao desempenho no processo de transmissão das informações considerando o modo como às informações são dispostas quanto à clareza, intuitividade e 
atratividade da ferramenta. A 'Comunicabilidade' foi tratada com relação ao conteúdo das informações - sobretudo quanto à orientação sobre horários, itinerários e confiabilidade do usuário no cumprimento do que é previsto - conforme Figura 2.

Figura 2 - Resultado do critério 'Interface'

\begin{tabular}{|c|c|c|c|}
\hline \multicolumn{4}{|l|}{200} \\
\hline 150 & \multicolumn{2}{|l|}{151} & 158 \\
\hline 100 & \multicolumn{2}{|l|}{100} & 83 \\
\hline 50 & 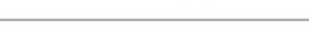 & & \\
\hline 0 & & & \\
\hline \multirow{2}{*}{-50} & \multirow{2}{*}{\multicolumn{2}{|c|}{-39}} & 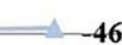 \\
\hline & & & \\
\hline-100 & $\begin{array}{l}\text { 2.1.Layout e forma de } \\
\text { apresentar }\end{array}$ & 2.2.Com unicabilidade & Global \\
\hline$\leadsto$ UsabTICMáx & 151 & 163 & 158 \\
\hline -E-UsabTIC & 100 & 72 & 83 \\
\hline --UsabTICMín & -39 & -52 & -46 \\
\hline
\end{tabular}

Fonte: Autoria própria (2016).

Nesse sentido, a questão da 'Comunicabilidade' obteve o pior desempenho na avaliação, de modo que a distância entre desempenhos percebido e máximo foi igual a 91. O subcritério 'Layout' obteve melhor desempenho em comparação à 'Comunicabilidade', apresentando uma distância de apenas 51 pontos do máximo desempenho. Nesse sentido, a maioria dos entrevistados - 38,7\% considera a questão da atratividade da ferramenta pouco significante para a compreensão de usabilidade das TIC.

Apesar de ser possível inferir que os usuários consideram que as TIC possuem um aspecto físico razoável de apresentação - estando dentro das expectativas, porém apresentando-se pouco relevante para a usabilidade do serviço -, de modo geral, elas não cumprem de maneira satisfatória sua função no sentido de prestação de informações - horário, itinerários, tempo de viagem, rota, etc.

Em uma análise global do critério, verificou-se uma maior distância entre os pontos mínimo e percebido - 129 - do que em relação ao ponto máximo - 75 - o que denota uma necessidade de melhoria de desempenho em quase $50 \%$.

\subsection{Conforto na movimentação do usuário}

Este aspecto está relacionado ao compromisso de assegurar ao usuário a possibilidade de deslocamento em menor tempo possível, tendo em vista a questão da infraestrutura (organização, horários e rotas).

Os questionamentos direcionados aos usuários foram baseados nos subcritérios: 'Satisfação' - no qual se procurou avaliar, por exemplo, questões relacionadas à percepção de autonomia do usuário e tempo de resposta do sistema; 'Agilidade e Tempo' - no qual se buscou avaliar questões 
como tempo de espera do veículo, entre o que é informado pelos totens e o que de fato acontece, e contribuição desse sistema de informações para a melhor gestão de tempo do usuário; 'Acesso/Acessibilidade' - no qual se verificou as questões relacionadas ao acesso aos pontos de aquisição e recarga de cartão e percepção de qualidade de integração de recursos audiovisuais voltados para atendimento de pessoas com deficiência auditiva ou visual, conforme demonstrado na Figura 3.

Figura 3 - Resultado do critério 'Conforto na movimentação do usuário'

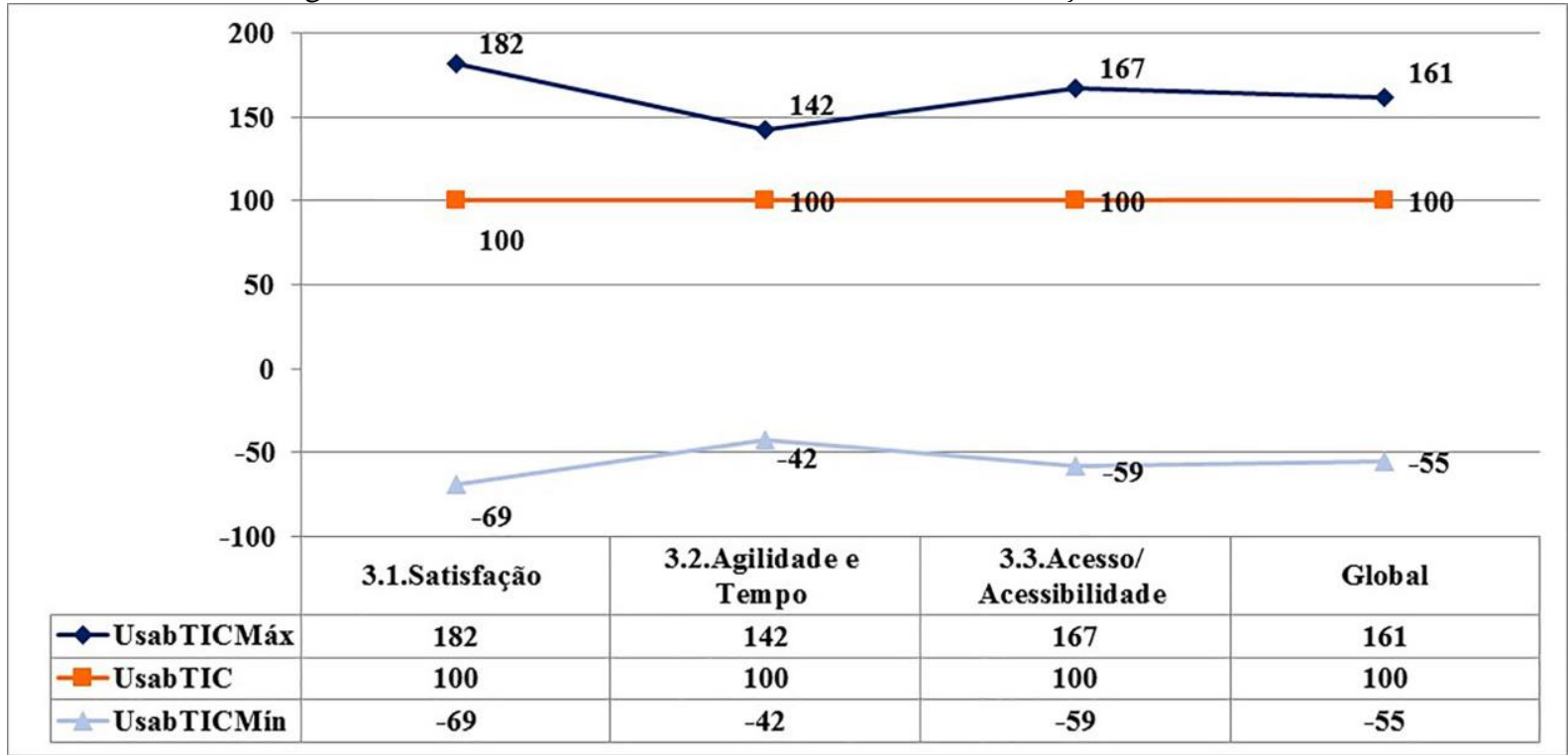

Fonte: Autoria própria (2016).

O melhor desempenho obtido dentre os subcritérios de análise foi 'Agilidade e tempo', apresentando uma distância de 42 pontos entre o nível de desempenho percebido e a máxima capacidade. O pior desempenho percebido refere-se a 'Satisfação', no qual a distância entre desempenho percebido e máximo é de 82.

Em uma análise global do critério, observou-se que, apesar de os usuários não se demonstrarem satisfeitos com relação às TIC e - apesar dos problemas existentes nos processos de transmissão da informação -, há o reconhecimento de que essas contribuem de maneira significativa para o conforto na movimentação do usuário.

\subsection{Valor da marca BRT}

De acordo com Feldwick (1996), o valor da marca estaria associado - em duas de suas classificações - ao conjunto de crenças e associações que o consumidor tem sobre a marca. Poderia estar associado ainda a uma medida de afeto/sentimento do consumidor com a marca, conforme demonstrado na Figura 4.

Figura 4 - Resultado do critério 'Valor da Marca BRT' 


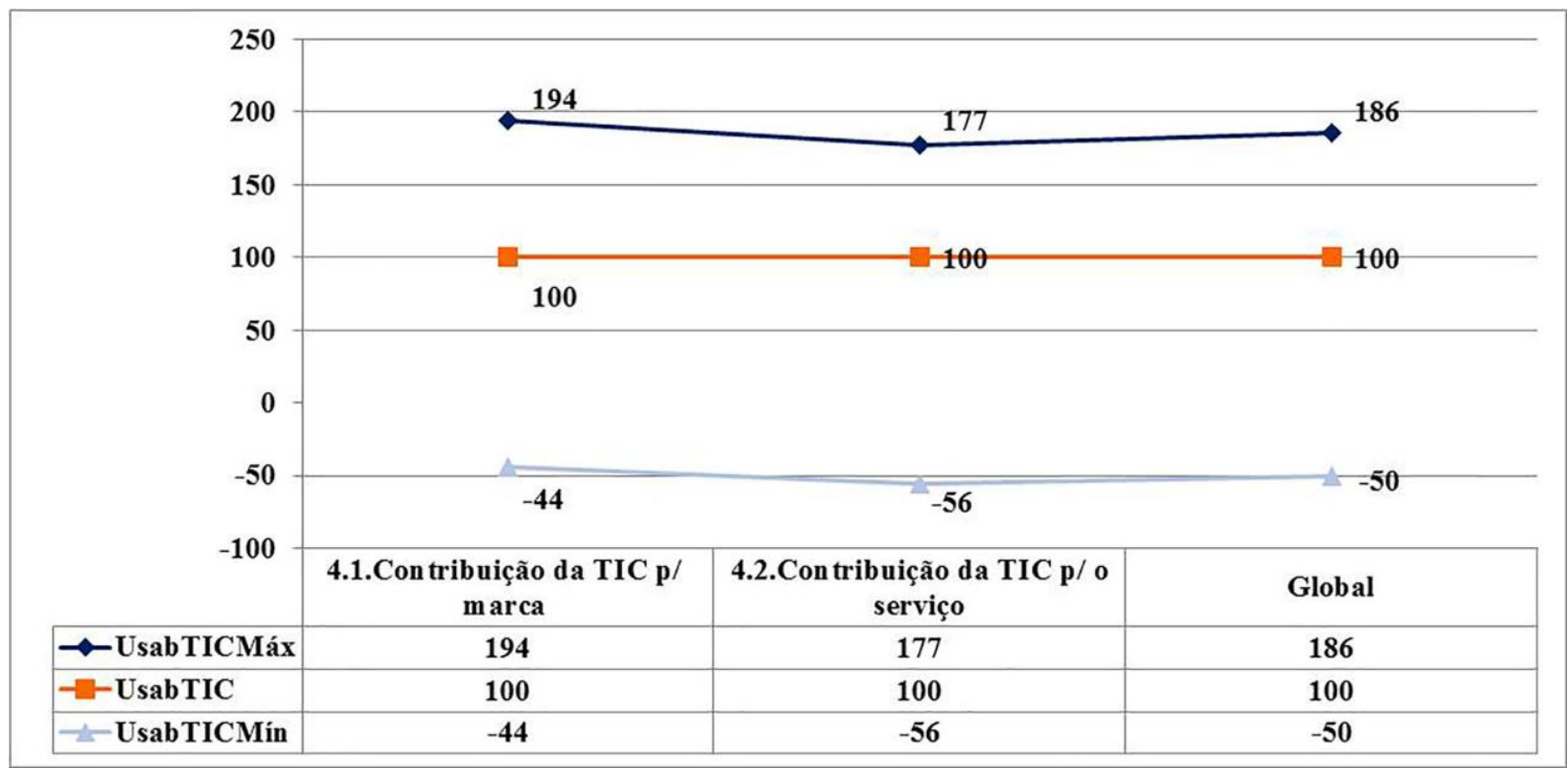

Fonte: Autoria própria (2016).

Nesse aspecto, inferiu-se que os usuários percebem a contribuição da utilização das TIC tanto para a marca quanto para o desempenho do BRT do mesmo modo - desempenho igual a 100. Em nível global, o critério apresentou um desempenho razoável, no qual a distância entre o desempenho percebido e máximo foi de 86.

\subsection{Análise de Usabilidade das TIC no sistema BRT}

Cybis et. al., (2010) afirma que a usabilidade em transportes poderia estar associada à capacidade que um sistema interativo tem para efetuar tarefas de modo eficiente, eficaz e agradável. A Figura 5 demonstra os resultados obtidos de modo geral.

Figura 5 - Resultado 'Usabilidade das TIC no sistema BRT'

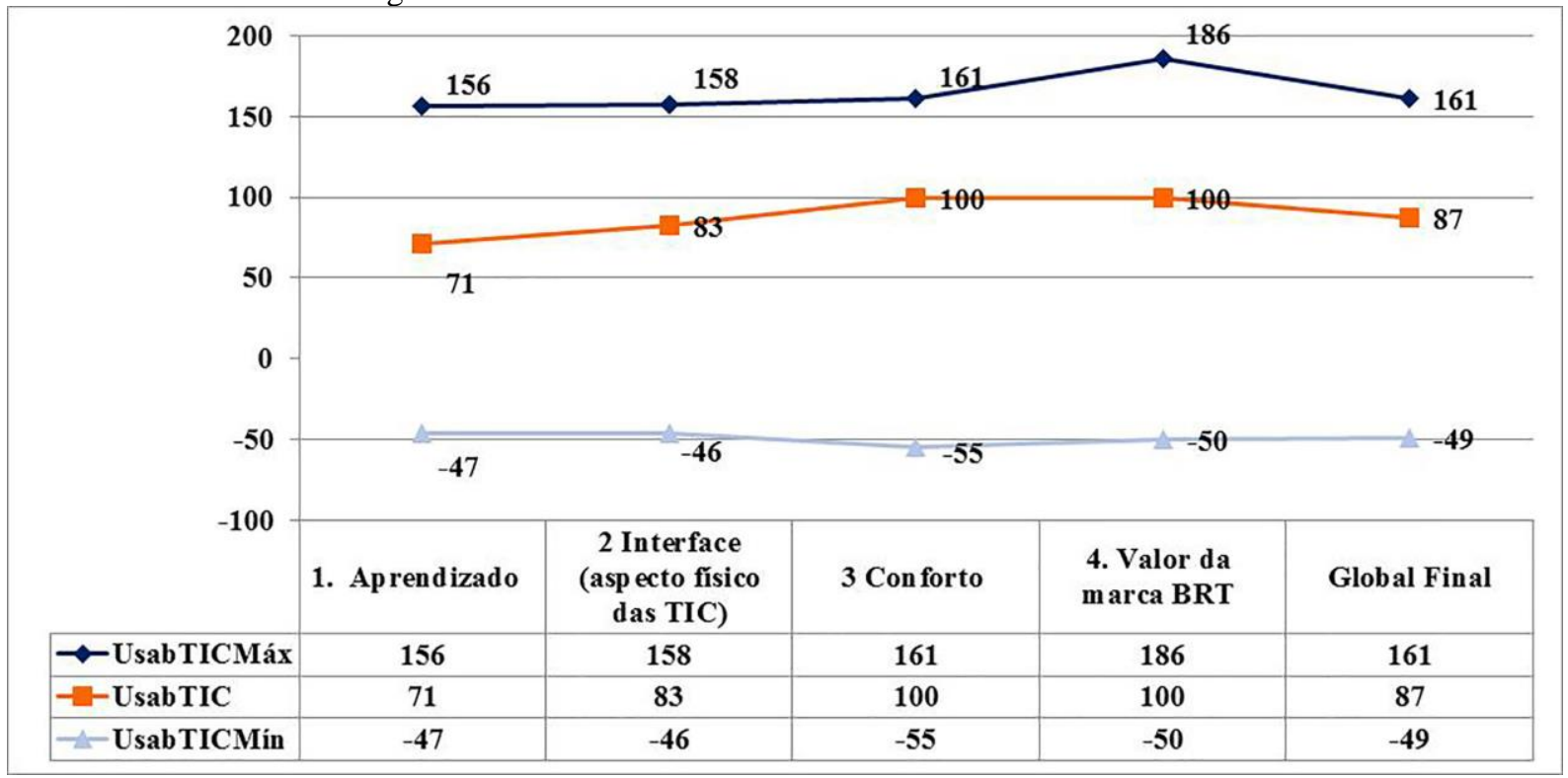

Fonte: Autoria própria (2016). 
A partir da análise gráfica, foi possível auferir que o critério conforto obteve melhor desempenho de usabilidade na avaliação dos usuários, enquanto o valor da marca obteve o pior resultado. Em relação ao conforto, é possível perceber uma menor distância entre os pontos de desempenho mínimo e percebido pelos usuários, o que sinaliza que, embora não se possa afirmar um estado de plena satisfação, infere-se que existe a percepção de contribuição das TIC para que o usuário obtenha maior autonomia na utilização do BRT.

$\mathrm{Na}$ questão do aprendizado - referente à capacidade de compreensão e retenção da informação - observou-se a existência de uma distância significativa entre nível percebido pelo usuário e o desempenho de usabilidade máximo. Este pode ser um indicativo de que as ferramentas de informação não têm cumprido com seu papel de informar de maneira suficientemente adequada.

Em uma percepção global da usabilidade das TIC no sistema BRT, depreende-se que os usuários consideram sua utilização de relevância para a qualidade do serviço prestado. De acordo com Shackel (1991), a usabilidade é o principal atributo para identificação da qualidade necessária para a aceitabilidade de um produto/ serviço no mercado.

O critério Conforto foi considerado o principal elemento de impacto para usabilidade do serviço. Os usuários atribuem maior valor a questões como percepção de autonomia e reconhecem a contribuição do sistema de informações para a melhor gestão de tempo. Os demais critérios Aprendizado e Interface - obtiveram desempenhos inferiores na avaliação de desempenho de usabilidade. Nesse sentido, os usuários atribuem pouca importância à atratividade da ferramenta e consideram que a função das TIC - comunicar - não está sendo desempenhada de maneira satisfatória.

Nielsen (1993) atribui à usabilidade a função de múltiplos componentes e define que essa é tradicionalmente associada a cinco atributos básicos: Facilidade, Eficiência, facilidade de relembrar, erros e satisfação subjetiva. Nesse sentido, a partir dos resultados obtidos, os usuários consideram o desempenho dentro das expectativas, quanto à facilidade de utilização, porém percebe-se o não cumprimento dos horários estabelecidos, de modo que não chega a comprometer de forma tão impactante a prestação do serviço de modo geral.

Desse modo, a utilização das TIC apresenta significância para o conceito de usabilidade do sistema BRT, mas se reconhece o não funcionamento em seu modo mais eficiente quanto poderia ser. Cybis et. al., (2010) afirma, nesse sentido, que a ocorrência de problemas no processo de interação homem-máquina pode desenvolver no usuário sensações como desapontamento, ansiedade e antipatia.

De acordo com Raia Junior (2000), é preciso estabelecer mecanismos de estímulo de modo que os usuários vivenciem novas experiências que os faça optar pela utilização de meios de transportes públicos ao invés de particulares. A utilização de dispositivos tecnológicos de 
comunicação em transportes tem se demonstrado de grande relevância nesse sentido. Como principal barreira ao avanço em sua utilização - conforme afirma Silva et al., (2015) - está a questão da não priorização dos gestores na aplicação de recursos nesse setor.

\section{Conclusões}

A partir desta análise, foi possível pensar questões voltadas para a compreensão de usabilidade na concepção dos usuários do sistema BRT Sul. A principal contribuição se refere à apresentação de um modelo capaz de prestar suporte informacional no processo de tomada de decisões, já que, diferentemente do que se percebe na visão tradicional Pesquisa Operacional - na qual se identifica apenas um ponto possível para a solução ótima -, é capaz de demonstrar resultados diversos e abrir um leque de possibilidades para implementação de soluções mais efetivas.

Os resultados apresentados demonstraram-se capazes de apoiar os gestores públicos/tomadores de decisão ao considerar o fato de que a aplicação do método permitiu sutilizar aspectos que, de fato, apresentam significância para a usabilidade do sistema na perspectiva do usuário. A vantagem disso se refere à possibilidade de aplicação dos recursos de modo mais eficiente possível, assumindo-se o fato de que esses são escassos.

Os objetivos propostos pela pesquisa foram alcançados, uma vez que foi possível identificar a percepção do usuário quanto à concepção de usabilidade das TIC no sistema BRT. A utilização do modelo MCDA-C deu respaldo para a consideração de opinião das diversas partes envolvidas ponto de vista dos especialistas (representantes dos decisores) - no processo de elaboração dos critérios e subcritérios.

Por fim, cabe considerar que, como qualquer modelo, o método MCDA apresenta limitações. Conforme afirma Silveira Jr. (2016), esta construção não tenciona solucionar todos os problemas relacionados ao processo decisório, mas indicar caminhos que sejam capazes de satisfazer o problema. Não necessariamente o melhor caminho - enfoque da Pesquisa Operacional tradicional -, tendo em vista que, ao tratar de múltiplos objetivos, torna-se impraticável a determinação de uma solução única.

\footnotetext{
Abstract

The urban transport system is considered a basic character of service to the population, as it ensures the user access to mobility. The BRT system has shown a more advantageous alternative compared to other transport systems due to lower complexity and cost of deployment. Information and Communication Technologies (ICT) in this sense plays an important role in not only the BRT, but any transport system to reach higher levels of efficiency in service delivery. Thus, this study aimed to analyze the perception of users with regard to ICT use in BRT South system - which serves as the administrative regions of Santa Maria, Gama and Park Way - from the multi-criteria analysis, which was intended to verify the influence of these for understanding of usability / quality of service. As a
} 
result, it was found that users consider their use of relevance to the quality of service, so the comfort criterion was considered the main impactor for usability of the service while learning and Interface had lower performances.

Key-words: usability; information and communication technologies (ICT); BRT.

\section{Referências}

ABRAHÃO, J. I.; SILVINO, A..M.D; SARMET, M.M. Ergonomia, Cognição e Trabalho Informatizado.

Psicologia: Teoria e Pesquisa. Mai-Ago 2005, v. 21, n. 2, p. 163-171, 2005. Disponível em: <

http://www.scielo.br/pdf/\%0D/ptp/v21n2/a06v21n2.pdf >. Acesso em: 10 mar. 2016.

ANDRADE, A. L. L. Usabilidade de interfaces WEB: avaliação heurística no jornalismo online. Rio de Janeiro: Epapers, 2007.

ARAÚJO, M.R.M.; OLIVEIRA, J. M.; JESUS, M. S.; SÁ, N. R.; SANTOS, P. A. C.; \& Lima, T. C. Transporte Público Coletivo: discutindo acessibilidade, mobilidade e qualidade de vida. Psicologia \& Sociedade, v. 23, n. 2, p. 574-582, 2011. Acesso em: < http://www.scielo.br/pdf/psoc/v23n3/15.pdf >. Disponível em: 12 mar. 2016.

BANA E COSTA, C.A. Multiple Criteria Decision Aid: An Overview. In: BANA E COSTA (ed.). Readings in Multiple Criteria Decision Aid. Berlin: Springer, 1990. p.1-4.

BARAT, J.; BATISTA, M.S.N. Transporte público e programas habitacionais. Pesquisa e Planejamento Econômico, n.3, 1973. p. 375-388.

BRT Centre of Excellence; EMBARQ; IEA e SIBRT. Global BRTdata: version 1.22, last modified on June 27, 2013. Disponível em: < http://www.brtdata.org >. Acesso em: 18 abr. 2016.

CYBIS, W.; BETIOL, A.; FAUST, R. Ergonomia e Usabilidade Conhecimentos, Métodos e Aplicações. 2. ed. São Paulo: Novatec, 2010. p. 352.

ENSSLIN, L.; NETO, G.M; NORONHA, S.M. Apoio à Decisão: Metodologias para Estruturação de Problemas e Avaliação Multicritério de Alternativas. Florianópolis: Insular, 2001. p. 296.

FELDWICK, P. Do we really need brand equity? The Journal of Brand Management. v. 4 (1), 1996. p. 9-28. crossref

FERREIRA, A. B. H. Aurélio século XXI: o dicionário da Língua Portuguesa. 3. ed. rev. e ampl. Rio de Janeiro: Nova Fronteira, 1999. p. 2128.

FERRIS, B. OneBusAway: Improving the Usability of Public Transit. Seattle, Washington, WI. (Doutorado). University of Washington, 2011. p. 234.

FLORES, L.C.S.; CAVALCANTE, L.S.; RAYE, R.L. Marketing turístico: Estudo sobre o uso da tecnologia da informação e comunicação nas agências de viagens e turismo de Balneário Camboriú (SC, Brasil). In: REVISTA BRASILEIRA DE PESQUISA EM TURISMO. v. 6, n. 3, 2012. p. 322-339. Disponível em: <

http://www.rbtur.org/rbtur/article/view/487 >. Acesso em: 13. mar. 2016. 
JORDAN, P. W. Human factors for pleasure in product use. Applied Ergonomics. v. 29(1), 1998. p. 25-33. Disponível em: < http://www.cse.chalmers.se/research/group/idc/ituniv/kurser/10/hcd/literatures/patrick\%20Jordan\%201998.pdf >. Acesso em: 10 mar. 2016.

KANNINEN, B. J. Intelligent Transportation Systems: an Economic and Environmental Policy Assessment. Transportation Research, Londres, v. 30 (1), 1996. p. 1-10. crossref

LEVINSON, H.; ZIMMERMAN, S.; CLINGER, J.; RUTHERFORD, S.; SMITH, R.L.; CRACKNELL, J.; \& SOBERMAN, R. Bus Rapid Transit, Volume 1: Case Studies in Bus Rapid Transit. Transit Cooperative Research Program (TCRP) Report 90, published by the Transportation Research Board, Washington, 2003.p. 3.

MINISTÉRIO DAS CIDADES. Manual de BRT - Bus Rapid Transit - Guia de Planejamento. Brasília: Ministério das Cidades, dez. 2009. p.182.

NIELSEN, J. Usability Engineering. Academic Press, Cambridge, MA, 1993.

NTU - Associação Nacional das Empresas de Transportes Urbanos. Avaliação comparativa das modalidades de transporte público urbano. Elaborado por Jaime Lerner Arquitetos Associados. Brasília, jul. 2009.

PDTU. Plano Diretor de Transporte Urbano e Mobilidade do Distrito Federal e Entorno. Secretaria de Estado de Transportes. Governo do Distrito Federal, 2010.

SAMPAIO, B.R.; NETO, O.L.; SAMPAIO, Y. Eficiência na Gestão do Transporte Público: Lições para o Planejamento Institucional. In: PLANEJAMENTO E POLÍTICAS PÚBLICAS, n. 29, 2006, p. 101-121. Disponível em: < http://www.ipea.gov.br/ppp/index.php/PPP/article/viewFile/45/44 >. Acesso em: 14 mar. 2016.

SILVA, R.B; SHIMISHI, J.M; RODRIGUES, E.C.C. Metodologia para Investigação da Percepção das Tecnologias de Informação e Comunicação (TIC) na Usabilidade do Sistema BRT/Sul de Brasília/DF. In: XXIX CONGRESSO

NACIONAL DE DE PESQUISA EM TRANSPORTE DA ANPET. 29, Ouro Preto. Ouro Preto, MG. 2922-2925, 2015.

SILVEIRA JR., A. Metodologia para avaliar as condições de operação do transporte de carga por cabotagem no Brasil, sob a ótica dos armadores. 2016. 234f. Dissertação (Doutorado em Transportes). Programa de Pós-Graduação em Transportes, Universidade de Brasília, Brasília, 2016.

SHACKEL, B. Usability - Contest, Framework, Definition, Design and Valuation. In: SHACKEL, B.; RICHARDSON, S. Human Factors for Informatics Usability. Cambridge: Academic Press, 1991. p. 21-33. Disponível em: < https://books.google.com.br/books?hl=ptBR\&lr=\&id=KSHrPgLlMJIC\&oi=fnd\&pg=PA21\&dq=Usability+\%E2\%80\%9 $3+$ context,+framework,+design+and+evaluation\&ots=IVPvOUYZGe\&sig=ulbjeTYE-

9czeuwHiv8RH8ecMF0\#v=onepage\&q\&f=true > Acesso em: 21 de Abril de 2015.

RAIA JUNIOR, A.A. Acessibilidade e Mobilidade na Estimativa de um Índice de Potencial de Viagens Utilizando Redes Neurais Artificiais e Sistema de Informações Geográfica. 2000. 212f. Dissertação (Doutorado em Engenharia Civil). Universidade de São Paulo, São Carlos, 2000. Disponível em: http://www.teses.usp.br/teses/disponiveis/18/1813 7/tde-10112001-160812/pt-br.php >. Acesso em: 26 mar. 2016.

REIS, J.G.M.; LIMA, J.O.; MACHADO, S.T. Bus Rapid Transit (BRT) como solução para o transporte público de passageiros na cidade de São Paulo. In: INOVAE - JOURNAL OF ENGINEERING AND TECHNOLOGY INNOVATION, v. 1 n. 1, 2013. p. 83- 98. 
RODRIGUES, E.C.C. Metodologia para Investigação da Percepção das Inovações na Usabilidade do Sistema Metroviário: Uma Abordagem Antropotecnológica. 2014. 262f. (Dissertação Doutorado em Transportes). Programa de Pós-Graduação em Transportes, Universidade de Brasília, 2014.

YAGER, R.R. On Ordered Weighted Averaging Aggregation Operators in Multicriteria Decision Making. IEEE Transactions on Systems, Man and Cybernetics, v. 18 (1), 1998. p. 183-190. crossref

ZANELLA, L.C.H. Quanto aos objetivos da Pesquisa. In: ZANELLA, L.C.H. Metodologia de Estudo e Pesquisa em Administração. Florianópolis: CAPES, Departamento de Ciências da Administração / UFSC; [Brasília]: UAB, 2009. p. 79.

\section{Dados dos Autores}

Nome completo: Fernanda Santos Lima

Filiação Institucional: Universidade de Brasília

Departamento: Administração

Função ou cargo ocupado: Graduada

Endereço completo para correspondência: Quadra 104 conjunto 10 casa 15,

Telefone para contato: (61) 99306-6748

E-mail:fslima26@gmail.com

\section{Nome completo: Evaldo Cesar Cavalcante Rodrigues}

Filiação Institucional: Universidade de Brasília

Departamento: de Administração - FACE

Função ou cargo ocupado: Professor Efetivo

Endereço completo para correspondência: Campus Darcy Ribeiro - Prédio da FACE - Asa Norte

- Brasília - DF - CEP: 70910-900

Telefone para contato: (61) 99300-6008

E-mail:evaldocesar@unb.br

Nome completo: Roberto Bernardo da Silva

Filiação Institucional: Universidade de Brasília

Departamento: Engenharia Civil e Ambiental - Programa de Pós-graduação em Transportes

Função ou cargo ocupado: Doutorando

Endereço completo para correspondência: Campus Universitário Darcy Ribeiro - Prédio SG 12 da FT - Brasília-DF | CEP 70910-900

Telefone para contato: (61) 98130-8586

E-mail: rbaccioly@gmail.com

\section{Nome completo: Carlos Rosano Peña}

Filiação Institucional: Universidade de Brasília 
Departamento: Administração

Função ou cargo ocupado: Professor Adjunto

Endereço completo para correspondência: Campus Darcy Ribeiro - Prédio da FACE - Asa Norte

- Brasília - DF - CEP: 70910-900

Telefone para contato: (62) 99299-9917

E-mail: gmcrosano@gmail.com

Submetido em: 14-12-2016

Aceito em: $31 / 12 / 2016$ 